\title{
Review Article \\ Providing Care in Emergency Department Hallways: Demands, Dangers, and Deaths
}

\author{
John R. Richards, ${ }^{1}$ M. Christien van der Linden, ${ }^{2}$ and Robert W. Derlet ${ }^{1}$ \\ ${ }^{1}$ Department of Emergency Medicine, U.C. Davis Medical Center, University of California, PSSB 2100, \\ 2315 Stockton Boulevard, Sacramento, CA 95817, USA \\ ${ }^{2}$ Accident and Emergency Department, Medical Center Haaglanden, The Hague, Netherlands \\ Correspondence should be addressed to John R. Richards; jrrichards@ucdavis.edu
}

Received 12 October 2014; Accepted 9 December 2014; Published 25 December 2014

Academic Editor: Frank Hildebrand

Copyright (C) 2014 John R. Richards et al. This is an open access article distributed under the Creative Commons Attribution License, which permits unrestricted use, distribution, and reproduction in any medium, provided the original work is properly cited.

\begin{abstract}
Emergency departments (ED) worldwide have experienced dramatic increases in crowding over the past 20 years that now have reached critical levels. One consequence of ED crowding has been the routine use of ED hallways for patient care. This includes ED patients who are awaiting care but are considered unstable to remain in the waiting room, patients who are undergoing active medical and trauma treatment, and patients who have been stabilized but await transfer to an inpatient bed (boarding) or another institution. Compared with licensed hospital or standard ED beds, care in ED hallways results in increased patient morbidity and mortality, as well as patient and staff dissatisfaction. Complications experienced by hallway patients include unrecognized sudden respiratory arrest or unstable cardiac arrhythmias, delay in time-sensitive procedures and laboratory testing, delay in receiving important medications, excessive or unrelieved pain, overall increased length of stay, increased disability, and exposure to traumatic psychological events. While much has been published on the general problems of ED crowding, only recently have studies focused exclusively on the issues of providing care in ED hallways. This review summarizes the current issues, challenges, and solutions for hallway care.
\end{abstract}

\section{Introduction}

Prior to the 1990s providing care in emergency department (ED) hallways was uncommon, occurring only periodically for short segments of time $[1,2]$. Influx of patients generally matched a corresponding outflow, either by discharge home or admission to inpatient units. In some EDs, empty beds were reserved to ensure adequate surge capacity for a sudden influx of patients. In the 1990s, crowding first became a concern in inner city and teaching hospital EDs [3, 4]. Over the next decade the majority of suburban and rural EDs would also experience crowded conditions $[5,6]$.

As crowding increased, the inflow of patients exceeded outflow for extended hours each day, resulting in the need to place patients somewhere [7]. For a growing number of EDs the solution was to move both stable and semistable patients from licensed ED beds into adjacent ED hallways, thus freeing up the official ED bed for another patient [8]. In these circumstances ED physicians face a difficult challenge. They must provide care to patients in the hallway with suboptimal nursing support and lack of privacy, which precludes a full history and physical examination. Patients may not be able to be fully monitored. Returning new patients back to the waiting room until a licensed ED bed becomes available poses a similar risk, as there is no way to directly observe or monitor patients. Patient care in ED hallways is fraught with delays and difficulties in laboratory testing, providing medication, supervising intravenous (IV) lines, recording vital signs, monitoring cardiac activity, or responding to deterioration in patient condition. The problem is further compounded when the ED physician has to simultaneously provide care to an excess number of patients in the hallway as well as in licensed ED bed spaces. Additional physicians and nurses are often not available to share the burden. In addition to risk of poor patient outcome, treating physicians are placed at increased risk for malpractice liability, medical 
board censure, or poor patient satisfaction survey results, which may adversely impact a physician's career [9].

\section{Who Gets Placed in ED Hallways and Why?}

The type of patient that is placed on a gurney in the ED hallway varies by institution and may vary within the same institution depending on who is in charge during times of crowding. Groups placed in hallways include (1) patients awaiting care but considered too unstable or ill to remain in the waiting room, (2) patients undergoing active medical and trauma care, but no vacant ED bed is available, (3) patients who are being "boarded" while awaiting transfer to an inpatient bed or transfer to another institution, (4) patients who arrive by ambulance but do not require immediate emergency care, and (5) patients awaiting transfer to a psychiatric facility $[5,8,9]$. Some states and nations have laws or rules regulating patient care in the $\mathrm{ED}$, requiring care in a licensed bed $[10,11]$. Other regulations forbid patient care out of sight and hearing of nurses and physicians, which is nearly impossible in crowded hallways [12]. We contend that routine care in ED hallways is illegal, unless the local authority licenses the beds, and mandated nursing, physician, and ancillary staffing are provided.

Emergency department hallway care may violate fire department codes [13]. Exceptions can be made only if hospital administration declares a disaster, which is a rare event. Disasters fall into two categories: external and internal. External disasters include airline crashes, shootings, and bombings, as well as chemical, radiation, and industrial accidents. Internal disasters result from situations at which hospital infrastructure is at risk, such as earthquakes, flooding, toxic material leak, loss of power, or hospital crowding (either the ED or inpatient wards). In theory, calling a disaster would provide additional resources so that patients could be moved out of crowded ED hallways. However, most hospitals are not equipped to be in frequent disaster mode, whereas many EDs have crowded hallways every day. Despite this, internal disasters are rarely called for this reason. The authors are personally aware of cases where ED physicians have been discouraged from calling internal disasters during periods of dangerous crowding and have been disciplined for doing so. In some states, the law establishes a standard patient to nurse ratio in the ED to enhance patient safety and prevent work overload to nursing staff [14]. However, these ratios can be circumvented by the use of selective patient designation and hallway care. For example, if 24 patients are in a hallway with only two nurses assigned to the hallway, only 8 might officially be counted as actual ED patients. The remaining 16 could be counted as waiting room patients, for whom the sole provider of nursing care may be the ED physician.

A progressive rise in patients treated in the hallways over years has resulted in the development of ad hoc labeling, with letters or numbers on the wall where gurneys are parked $[7,8]$. Recently, placement of patients needing transfer to inpatient psychiatric units in other hospitals has become a problem [15]. These patients may lie on gurneys in ED hallways for many days without privacy, readily accessible bathroom facilities, showers or baths, scheduled medications, and meals. It is difficult to believe this is legal in the modern era. It may appear that government officials, hospital administrators, and licensing agencies are not fully addressing the legal implications of ED boarding. The authors are personally aware of a recent Joint Commission inspection of an ED in the USA. Rather than citing the hospital after walking down hallways crowded with patients in acute discomfort, the inspectors only cited the hospital because an ED refrigerator was set at the wrong temperature. The Joint Commission has recognized the problem of hallway care only recently and in 2014 created a standard that limits boarding of inpatients to no longer than 4 hours [12].

\section{Does Hallway Care Occur Worldwide?}

Care in ED hallways is an international problem. The problem of increasing patient flow into an overburdened system has been described in Asia, the Middle East, Canada, Australia, New Zealand, South America, and Europe [16-23]. The primary reasons for the use of hallways for patients are similar to the United States: (1) work-up of new patients and (2) boarding of patients admitted to inpatient units. Crowded public and teaching hospitals are most severely impacted, whereas use of hallways for ED care is less common in private hospitals. Unlike the USA, where the Emergency Medical Treatment \& Labor Act (EMTALA) requires that nearly all hospitals stabilize patients with emergency conditions, most other counties do not have laws mandating ED care be given in private hospitals if patients have no means of paying for their care. Therefore private hospitals may not experience hallway crowding, whereas their public counterparts may be required to evaluate every person who presents to the ED. Public hospitals in parts of Asia and South American have reported that patients may lay on hallway gurneys, unattended for over 24 hours. Some countries have adapted policies in an attempt to limit hallway crowding. For example, in England, a new policy was introduced in the early 2000s mandating patient admission or discharge home within 4 hours of patient presentation to the ED [24].

\section{Poor Outcomes from Hallway Care}

The growing list of published studies unanimously concludes that the quality of ED care decreases as crowding increases, in part from hallway care. Medical malpractice actions have occurred as a result of patients receiving care in hallways [9]. There are several studies on the outcome of boarding admitted inpatients in EDs [25-32]. While ED boarding is not synonymous with hallway care, boarded patients in many hospitals spend a significant portion of their total time residing in hallways. Negative effects include death, preventable disability, prolonged hospital stays, discomfort, and dissatisfaction by both patients and staff [33-36]. The reasons are multifactorial. As noted above, absence of consistent monitoring is an important etiology. Patients who suddenly become unstable may be not recognized until it is too late. Patients have been found apneic, unconscious, or in shock laying unmonitored on hallway gurneys [37]. 
Nursing care is fragmented and inconsistent. In fact patients may linger in hallways without an assigned nurse to monitor and provide care [38-40]. Intravenous lines run dry or may become disconnected, risking air embolus. Delays or errors may occur in delivery of medications [41-44]. Pain is inadequately treated $[45,46]$. Patient "mix-ups" may occur when nursing and transportation staff is unfamiliar with the mapping of the hallway gurney spaces. Family members may not be able to visit patients due to space constraints. Coordination of care and communication is difficult in an ED hallway between ED physicians, physician assistants and nurse practitioners, admitting and consulting physicians, nurses, social workers, and family members. Patients awaiting placement in inpatient psychiatric facilities may have their problems compounded by exposure, lack of privacy, food, and bathroom facilities for days before being removed from hallway gurneys [47]. The electronic medical record (EMR) may make matters worse for hallway patients, as they may appear in separate, hidden screens and may be moved to different locations frequently. The authors are personally aware of cases where these patients have been erroneously discharged, reported as "left without being seen," or "eloped" from the EMR. Delays in initiating admission treatment orders may result from software systems that do not recognize that patients are boarded in the hallway.

Morbidity and mortality as a result of hallway care may be underreported. Although these events are reviewed by ED continuous quality and performance improvement committees, physicians may be discouraged from publishing the data because it will reflect negatively on the institution. This is especially true in nonuniversity hospitals, where academic freedom is not guaranteed. Delays resulting from ED hallway care leading to patient death have been reported [25-32]. The lay press periodically publishes anecdotes of deaths that occur in ED areas not designated for actual patient care $[48,49]$. The authors believe that the public is not fully aware of the morbidity and mortality that result from hallway care.

\section{Solutions to Hallway Care}

The obvious solution to reducing and eventually ending the practice of hallway care is to solve the overall ED crowding problem. Several professional societies including Society of Academic Emergency Medicine (SAEM), American College of Emergency Physicians (ACEP), American Academy of Emergency Physicians (AAEM), Emergency Nurses Association (ENA), College of Emergency Medicine (CEM), Australasian College of Emergency Medicine (ACEM), Canadian Association of Emergency Physicians (CAEP), and the Institute of Medicine (IOM) have published proposals to reduce or end ED crowding [50-57]. The prospect of reducing ED crowding in the near future may be elusive, as many EDs have made major attempts for the past two decades with minimal or no improvement $[58,59]$. Simply building a larger ED to increase capacitance may not solve the problem, as the chief reason for overcrowding is "exit block," or the impaired ability to move patients out of the $\operatorname{ED}[60,61]$. However, a focused approach to solve hallway crowding has been adapted by leading hospitals [62, 63]. Emergency and inpatient physicians at the Stony Brook University Hospital in New York collaborated and developed a program whereby admitted hallway patients are transferred out of the ED to inpatient hallways [64]. Patients receive more attention in a quieter and more private environment. This transfer of patient care has been shown to be safe, and patients prefer being boarded in inpatient hallways [65-67]. Nurses prefer it as well. In a survey of both ED and inpatient nurses on the concept, inpatient nurses stated their preference that patients remained in the $\mathrm{ED}$, but when asked if they themselves were the patient, they indicated the preference to be boarded in an inpatient hallway [68]. Emergency department nurses preferred inpatient hallway boarding for patients and themselves if they were patients.

Other approaches to reducing the hallway problem have been more problematic. A common practice in some EDs has been to assign two patients into a single licensed ED bed space or exam room. These patients are then more immediately visible to ED staff and hence better monitored. But this is not a long-term solution, as "doubled up" patients still face privacy issues, physical crowding, infection cross-contamination, and compromises in care from understaffing. Additional solutions include adding more nursing and ancillary staff, streamlining the triage process, transferring patients to flexible areas of the hospital such as annexes, postanesthesia recovery units, or closed wards, or developing a limited "internal disaster" protocol $[69,70]$. The University of Southern California Medical Center in Los Angeles provides an example of a hospital that has developed a limited disaster protocol and surge plan [71]. Other solutions include posting waiting times for EDs online, allowing patients to check in online and wait at home for a telephone call or text message when an ED space is available, or to alert patients that hospital beds are filled and that boarding will be likely at the hospital, thus allowing them to choose to be seen elsewhere $[72,73]$.

Hospital leadership administrators have the system-wide power to make the changes necessary to decrease the clinical use of ED hallways. However, the response by hospital administration to ED hallway care varies widely by institution. Lack of available inpatient beds is the most common cause of ED crowding and patient boarding. Efficient and timely discharge of inpatients through the use of dedicated discharge suites, rapid postdischarge room cleaning, and cooperation of inpatient nurses to receive a new patient from the ED can only be achieved by hospital leadership mandate. Unfortunately many non-ED staff within the hospital believes the problem should remain in the ED. Some hospital administrators insist that care in ED hallways be provided but fail to provide logistical support needed to accomplish this task. Some ED staffing groups also incentivize ED physicians to evaluate patients in unlicensed areas by emphasizing metrics such as patients seen per hour. Physicians working for such groups may be censured or terminated for voicing opposition. These staffing groups must be educated to understand that better care for patients is also better for the group and institution.

There are several publications regarding loss of hospital revenue from ED crowding [74-78]. Further studies are needed to show hospital leadership that revenue declines with ED hallway care and boarding because patient throughput 
is adversely impacted, liability and malpractice exposure increases, and cases of preventable patient deaths rise. Dissatisfied patients may seek treatment at another hospital in the future and may share their negative experience with others, often through social media. The deleterious impact of ED hallway care on hospital revenue and patient safety may be the key factors to ultimately change the current philosophy, as there currently appears to be insufficient incentive to change. When a problem becomes "personal" and affects those in government, the press, or hospital administration, this incentive may accelerate in urgency [79]. Past examples of this are the development of Advanced Trauma Life Support (ATLS) and standardized trauma care and restrictions on resident working hours from the Libby Zion case [80, 81]. Proposed solutions which have been previously published can be summarized as follows.

Specific Solutions for Emergency Department (ED) Crowding, Patient Boarding, and Hallway Care

\section{Emergency Department}

(i) Improved triage efficiency and speed.

(ii) Bedside registration.

(iii) Increased nursing and technician staff during highvolume periods.

(iv) Increased physician coverage during high-volume periods.

(v) Expansion of ED space and number of beds.

(vi) Expansion of fast track, mid-track, and urgent care services.

(vii) Use of chairs instead of beds for patients who can sit to maximize space.

(viii) Observation unit for short-stay ED patients.

(ix) Physician-in-triage for rapid disposition.

(x) Selective imaging and laboratory testing performed in waiting room.

(xi) Triage of patients to nearby clinic after medical screening exam.

(xii) ED flow coordinator.

(xiii) Faster radiology and laboratory services, point-ofcare testing.

(xiv) Faster consultant response time, admission orders.

(xv) More efficient and faster discharge process of ED patients.

(xvi) "Holding orders" written by emergency physicians when inpatient bed is available.

(xvii) Noncritical imaging and laboratory testing deferred to inpatient or clinic setting.

(xviii) Ambulance diversion during times of ED crowding if possible.

(xix) Online scheduling of ambulatory ED visits. (xx) Real-time status of ED waiting times broadcast in waiting room.

(xxi) "Internal disaster" or similar ad hoc temporary crowding protocols.

(xxii) Telemedicine consults for potential ED-to-ED transfers.

(xxiii) Transfer of boarded patients to another hospital with available inpatient beds.

Inpatient

(i) Streamlined discharge process of inpatients.

(ii) Creation of a "discharge suite" for inpatients awaiting discharge.

(iii) Faster postdischarge inpatient room cleaning.

(iv) Inpatient hallway boarding.

(v) Use of ancillary space (recovery rooms, storage space, etc.) for inpatients.

(vi) Increased inpatient nursing staff during periods of ED crowding.

(vii) Cancellation of elective, nonurgent surgeries when hospital is full.

(viii) Inpatient flow coordinator.

(ix) Inpatient full capacity protocols.

\section{Nationwide}

(i) Improved access to primary care.

(ii) Increased number of primary care practitioners and clinics.

(iii) Extended hours and weekend availability for primary care clinics.

(iv) Increased reimbursement and salaries for primary care practitioners.

(v) Tuition assistance and loan forgiveness for students entering primary care.

(vi) Improved access to mental health facilities.

(vii) Increased number of mental health facilities and practitioners.

(viii) Increased reimbursement for mental health care.

(ix) Increased paramedic on-scene triage responsibility to prevent unnecessary transport.

\section{Conclusion}

Patients should not be routinely evaluated and treated in ED hallways where care is inferior. Hallway care has resulted in ED patient deaths and other poor outcomes. Options exist to eliminate ED hallway care and boarding but require initiative and support from hospital leadership to work collaboratively with nurses, physicians, and other healthcare providers. Such options should be aggressively pursued at all levels of the hospital. 


\section{Conflict of Interests}

The authors declare that there is no conflict of interests regarding the publication of this paper.

\section{Acknowledgment}

The authors would like to thank Dr. John Maa for critical analysis and revision of the paper.

\section{References}

[1] M. Nordberg, "Overcrowding: the ED's newest predicament," Emergency Medical Services, vol. 19, no. 4, pp. 33-44, 1990.

[2] R. W. Derlet and J. R. Richards, "Overcrowding in the nation's emergency departments: complex causes and disturbing effects," Annals of Emergency Medicine, vol. 35, no. 1, pp. 6368, 2000.

[3] J. R. Richards, M. L. Navarro, and R. W. Derlet, "Survey of directors of emergency departments in California on overcrowding," The Western Journal of Medicine, vol. 172, no. 6, pp. 385-388, 2000.

[4] R. W. Derlet, J. R. Richards, and R. L. Kravitz, "Frequent overcrowding in U.S. emergency departments," Academic Emergency Medicine, vol. 8, no. 2, pp. 151-155, 2001.

[5] J. C. Moskop, D. P. Sklar, J. M. Geiderman, R. M. Schears, and K. J. Bookman, "Emergency department crowding, part 1concept, causes, and moral consequences," Annals of Emergency Medicine, vol. 53, no. 5, pp. 605-611, 2009.

[6] L. I. Horwitz and E. H. Bradley, "Percentage of US emergency department patients seen within the recommended triage time: 1997 to 2006," Archives of Internal Medicine, vol. 169, no. 20, pp. 1857-1865, 2009.

[7] C. McNaughton, W. H. Self, I. D. Jones et al., "ED crowding and the use of nontraditional beds," American Journal of Emergency Medicine, vol. 30, no. 8, pp. 1474-1480, 2012.

[8] J. Freeman, "The emerging subspecialty of Hallway Medicine," Canadian Journal of Emergency Medicine, vol. 5, pp. 283-285, 2003.

[9] R. W. Derlet, R. M. McNamara, A. A. Kazzi, and J. R. Richards, "Emergency department crowding and loss of medical licensure: a new risk of patient care in hallways," The Western Journal of Emergency Medicine, vol. 15, no. 2, pp. 137-141, 2014.

[10] Department of Health \& Human Services (DHHS) and Centers for Medicare \& Medicaid Services (CMS), "CMS Manual System Pub 100-07,” 2008, https://www.cms.gov/Regulations-andGuidance/Guidance/Transmittals/downloads/R34SOMA.pdf.

[11] Wet op de Geneeskundige Behandelings Overeenkomst (WGBO) (boek 7, titel 7, afdeling 5 burgerlijk wetboek), 2014, http://wetten.overheid.nl/BWBR0007850/geldigheidsdatum_ 25-06-2014.

[12] Joint Commission on Accreditation of Healthcare Organizations (JCAHO), 2012, http://www.jointcommission.org/assets/ 1/6/Stds_Rev_Patient_Flow.pdf.

[13] National Fire Protection Association (NFPA), 2013, http://www. nfpa.org/newsandpublications/nfpa-journal/2013/january-february-2013/the-experts/in-compliance.

[14] T. C. Chan, J. P. Killeen, G. M. Vilke, J. B. Marshall, and E. M. Castillo, "Effect of mandated nurse-patient ratios on patient wait time and care time in the emergency department," Academic Emergency Medicine, vol. 17, no. 5, pp. 545-552, 2010.
[15] J. M. Nolan, C. Fee, B. A. Cooper, S. H. Rankin, and M. A. Blegen, "Psychiatric boarding incidence, duration, and associated factors in United States emergency departments," Journal of Emergency Nursing, 2014.

[16] J. M. Pines, J. A. Hilton, E. J. Weber et al., "International perspectives on emergency department crowding," Academic Emergency Medicine, vol. 18, no. 12, pp. 1358-1370, 2011.

[17] C. van der Linden, R. Reijnen, R. W. Derlet et al., "Emergency department crowding in the Netherlands: managers' experiences," International Journal of Emergency Medicine, vol. 6, no. 1, article 41, 2013.

[18] L. G. Stead, A. Jain, and W. W. Decker, "Emergency department over-crowding: a global perspective," International Journal of Emergency Medicine, vol. 2, no. 3, pp. 133-134, 2009.

[19] F.-Y. Shih, M. Huei-Ming, S.-C. Chen et al., "ED overcrowding in Taiwan: facts and strategies," The American Journal of Emergency Medicine, vol. 17, no. 2, pp. 198-202, 1999.

[20] H. Piene, "Hallway patients-a phenomenon possible to prevent?" Tidsskrift for den Norske Laegeforening, vol. 120, no. 22, p. 2616, 2000.

[21] M. Ardagh and S. Richardson, "Emergency department overcrowding - can we fix it?” The New Zealand Medical Journal, vol. 117, no. 1189, p. U774, 2004.

[22] K. Danielsen, A. M. Garratt, Ø. A. Bjertnæs, and K. I. Pettersen, "Patient experiences in relation to respondent and health service delivery characteristics: a survey of 26,938 patients attending 62 hospitals throughout Norway," Scandinavian Journal of Public Health, vol. 35, no. 1, pp. 70-77, 2007.

[23] R. Forero, K. M. Hillman, S. McCarthy, D. M. Fatovich, A. P. Joseph, and D. B. Richardson, "Access block and ED overcrowding," Emergency Medicine Australasia, vol. 22, no. 2, pp. 119-135, 2010.

[24] S. Mason, E. J. Weber, J. Coster, J. Freeman, and T. Locker, “Time patients spend in the emergency department: England's 4-hour rule-a case of hitting the target but missing the point?" Annals of Emergency Medicine, vol. 59, no. 5, pp. 341-349, 2012.

[25] D. B. Richardson, "Increase in patient mortality at 10 days associated with emergency department overcrowding," Medical Journal of Australia, vol. 184, no. 5, pp. 213-216, 2006.

[26] W. C. Cha, S. D. Shin, J. S. Cho, K. J. Song, A. J. Singer, and Y. H. Kwak, "The association between crowding and mortality in admitted pediatric patients from mixed adult-pediatric emergency departments in Korea," Pediatric Emergency Care, vol. 27, no. 12, pp. 1136-1141, 2011.

[27] E. J. Carter, S. M. Pouch, and E. L. Larson, “The relationship between emergency department crowding and patient outcomes: a systematic review," Journal of Nursing Scholarship, vol. 46, no. 2, pp. 106-115, 2014.

[28] K. D. Johnson and C. Winkelman, "The effect of emergency department crowding on patient outcomes: a literature review," Advanced Emergency Nursing Journal, vol. 33, no. 1, pp. 39-54, 2011.

[29] B. C. Sun, R. Y. Hsia, R. E. Weiss et al., "Effect of emergency department crowding on outcomes of admitted patients," Annals of Emergency Medicine, vol. 61, no. 6, pp. 605-e6, 2013.

[30] M. L. McCarthy, S. L. Zeger, R. Ding et al., "Crowding delays treatment and lengthens emergency department length of stay, even among high-acuity patients," Annals of Emergency Medicine, vol. 54, no. 4, pp. 492.e4-503.e4, 2009.

[31] A. Guttmann, M. J. Schull, M. J. Vermeulen, and T. A. Stukel, "Association between waiting times and short term mortality 
and hospital admission after departure from emergency department: population based cohort study from Ontario, Canada," British Medical Journal, vol. 342, no. 7809, Article ID d2983, 2011.

[32] S. Jo, Y. H. Jin, J. B. Lee, T. Jeong, J. Yoon, and B. Park, "Emergency department occupancy ratio is associated with increased early mortality," Journal of Emergency Medicine, vol. 46, no. 2, pp. 241-249, 2014.

[33] M. Walsh and J. C. Knott, "Satisfaction with the emergency department environment decreases with length of stay," Emergency Medicine Journal, vol. 27, no. 11, pp. 821-828, 2010.

[34] Y.-K. Lin and C.-J. Lin, "Factors predicting patients' perception of privacy and satisfaction for emergency care," Emergency Medicine Journal, vol. 28, no. 7, pp. 604-608, 2011.

[35] J. M. Pines, S. Iyer, M. Disbot, J. E. Hollander, F. S. Shofer, and E. M. Datner, "The effect of emergency department crowding on patient satisfaction for admitted patients," Academic Emergency Medicine, vol. 15, no. 9, pp. 825-831, 2008.

[36] J. C. Olsen and B. R. Sabin, "Emergency department patient perceptions of privacy and confidentiality," The Journal of Emergency Medicine, vol. 25, no. 3, pp. 329-333, 2003.

[37] K. J. Hong, S. D. Shin, K. J. Song, W. C. Cha, and J. S. Cho, "Association between ED crowding and delay in resuscitation effort," The American Journal of Emergency Medicine, vol. 31, no. 3, pp. 509-515, 2013.

[38] E. B. Kulstad and K. M. Kelley, "Overcrowding is associated with delays in percutaneous coronary intervention for acute myocardial infarction," International Journal of Emergency Medicine, vol. 2, no. 3, pp. 149-154, 2009.

[39] N. A. Bickell, U. Hwang, R. M. Anderson, M. Rojas, and C. L. Barsky, "What affects time to care in emergency room appendicitis patients?” Medical Care, vol. 46, no. 4, pp. 417-422, 2008.

[40] M. J. Schull, M. Vermeulen, G. Slaughter, L. Morrison, and P. Daly, "Emergency department crowding and thrombolysis delays in acute myocardial infarction," Annals of Emergency Medicine, vol. 44, no. 6, pp. 577-585, 2004.

[41] C. Fee, E. J. Weber, C. A. Maak, and P. Bacchetti, "Effect of emergency department crowding on time to antibiotics in patients admitted with community-acquired pneumonia," Annals of Emergency Medicine, vol. 50, no. 5, pp. 501.el-509.el, 2007.

[42] J. M. Pines, J. E. Hollander, A. R. Localio, and J. P. Metlay, "The association between emergency department crowding and hospital performance on antibiotic timing for pneumonia and percutaneous intervention for myocardial infarction," Academic Emergency Medicine, vol. 13, no. 8, pp. 873-878, 2006.

[43] E. B. Kulstad, R. Sikka, R. T. Sweis, K. M. Kelley, and K. H. Rzechula, "ED overcrowding is associated with an increased frequency of medication errors," The American Journal of Emergency Medicine, vol. 28, no. 3, pp. 304-309, 2010.

[44] R. Sikka, S. Mehta, C. Kaucky, and E. B. Kulstad, "ED crowding is associated with an increased time to pneumonia treatment," American Journal of Emergency Medicine, vol. 28, no. 7, pp. 809812, 2010.

[45] J. M. Pines and J. E. Hollander, "Emergency department crowding is associated with poor care for patients with severe pain," Annals of Emergency Medicine, vol. 51, no. 1, pp. 1-5, 2008.

[46] U. Hwang, L. D. Richardson, T. O. Sonuyi, and R. S. Morrison, "The effect of emergency department crowding on the management of pain in older adults with hip fracture," Journal of the American Geriatrics Society, vol. 54, no. 2, pp. 270-275, 2006.
[47] B. Kutscher, "Bedding, not boarding. Psychiatric patients boarded in hospital EDs create crisis for patient care and hospital finances," Modern Healthcare, vol. 43, no. 46, pp. 15-17, 2013.

[48] D. Holmberg, “The patients in the hallways," New York Times, 2007, http://www.nytimes.com/2007/06/10/nyregion/nyregionspecial2/10Rhospitals.html?fta=y\&_r=1\&.

[49] N. Corbett, "20-year-old patient dies in Abbotsford hospital hallway," Abbotsford News, January 2012, http://www .abbynews.com/news/136826343.html.

[50] "Ethics of emergency department triage: SAEM position statement. SAEM Ethics Committee (Society for Academic Emergency Medicine)," Academic Emergency Medicine, vol. 2, no. 11, pp. 990-995, 1995.

[51] American College of Emergency Physicians, Emergency Department Crowding: High-Impact Solutions, 2008, https://www.acep .org/workarea/DownloadAsset.aspx?id=50026.

[52] American Academy of Emergency Physicians, Position Statement on Emergency Department Crowding, 2005, http://www .aaem.org/em-resources/position-statements/2005/crowding.

[53] Emergency Nurses Association, "Emergency Nurses Association position statement: crowding in the emergency department," Journal of Emergency Nursing, vol. 32, no. 1, pp. 42-47, 2006.

[54] College of Emergency Medicine, Crowding in Emergency Departments, College of Emergency Medicine, London, UK, 2014, http://secure.collemergencymed.ac.uk/code/document .asp? ID=6296.

[55] Australasian College for Emergency Medicine, "Policy document-standard terminology," Emergency Medicine (Fremantle), vol. 14, pp. 337-340, 2002.

[56] Canadian Association of Emergency Physicians; National Emergency Nurses Affiliation, "Joint Position Statement on emergency department overcrowding," Canadian Association of Emergency Physicians, vol. 3, no. 2, pp. 82-88, 2001.

[57] Institute of Medicine, Hospital-Based Emergency Care: At the Breaking Point, 2006, http://www.iom.edu/reports/2006/ hospital-based-emergency-care-at-the-breaking-point.aspx.

[58] R. W. Derlet and J. R. Richards, "Ten solutions for emergency department crowding," The Western Journal of Emergency Medicine, vol. 9, pp. 24-27, 2008.

[59] A. Boyle, K. Beniuk, I. Higginson, and P. Atkinson, "Emergency department crowding: time for interventions and policy evaluations," Emergency Medicine International, vol. 2012, Article ID 838610, 8 pages, 2012.

[60] B. E. Mumma, J. Y. McCue, C.-S. Li, and J. F. Holmes, "Effects of emergency department expansion on emergency department patient flow," Academic Emergency Medicine, vol. 21, no. 5, pp. 504-509, 2014.

[61] S. G. Campbell and D. E. Sinclair, "Strategies for managing a busy emergency department," Canadian Journal of Emergency Medicine, vol. 6, no. 4, pp. 271-276, 2004.

[62] C. C. Won, D. S. Sang, J. S. Kyoung, K. J. Sung, and J. S. Gil, "Effect of an independent-capacity protocol on overcrowding in an urban emergency department," Academic Emergency Medicine, vol. 16, no. 12, pp. 1277-1283, 2009.

[63] S. K. Richardson, M. Ardagh, and P. Gee, "Emergency department overcrowding: the Emergency Department Cardiac Analogy Model (EDCAM)," Accident and Emergency Nursing, vol. 13, no. 1, pp. 18-23, 2005. 
[64] A. Viccellio, C. Santora, A. J. Singer, H. C. Thode Jr., and M. C. Henry, "The association between transfer of emergency department boarders to inpatient hallways and mortality: a 4year experience," Annals of Emergency Medicine, vol. 54, no. 4, pp. 487-491, 2009.

[65] P. Viccellio, J. A. Zito, V. Sayage et al., "Patients overwhelmingly prefer inpatient boarding to emergency department boarding," Journal of Emergency Medicine, vol. 45, no. 6, pp. 942-946, 2013.

[66] J. R. Richards, G. Ozery, M. Notash, P. E. Sokolove, R. W. Derlet, and E. A. Panacek, "Patients prefer boarding in inpatient hallways: correlation with the national emergency department overcrowding score," Emergency Medicine International, vol. 2011, Article ID 840459, 4 pages, 2011.

[67] P. Walsh, V. Cortez, and H. Bhakta, "Patients would prefer ward to emergency department boarding while awaiting an inpatient bed," Journal of Emergency Medicine, vol. 34, no. 2, pp. 221-226, 2008.

[68] B. C. Pulliam, M. Y. Liao, T. M. Geissler, and J. R. Richards, "Comparison between emergency department and inpatient nurses' perceptions of boarding of admitted patients," Western Journal of Emergency Medicine, vol. 14, no. 2, pp. 90-95, 2013.

[69] O. A. Soremekun, F. S. Shofer, D. Grasso, A. M. Mills, J. Moore, and E. M. Datner, "The effect of an emergency department dedicated midtrack area on patient flow," Academic Emergency Medicine, vol. 21, no. 4, pp. 434-439, 2014.

[70] D. R. Eitel, S. E. Rudkin, M. A. Malvehy, J. P. Killeen, and J. M. Pines, "Improving service quality by understanding emergency department flow: a white paper and position statement prepared for the American Academy of Emergency Medicine," The Journal of Emergency Medicine, vol. 38, no. 1, pp. 70-79, 2010.

[71] Los Angeles County, University of Southern California, 2007, http://www.calena.us/Resources/LAC\%20USC\%20Medical\% 20Center\%20Surge\%20Plan.pdf.

[72] S. W. Liu, A. G. Hamedani, D. F. M. Brown, B. Asplin, and C. A. Camargo Jr., "Established and novel initiatives to reduce crowding in emergency departments," Western Journal of Emergency Medicine, vol. 14, no. 2, pp. 85-89, 2013.

[73] A. Yip, S. McLeod, A. McRae, and B. Xie, "Influence of publicly available online wait time data on emergency department choice in patients with non critical complaints," Canadian Journal of Emergency Medicine, vol. 14, no. 4, pp. 233-242, 2012.

[74] T. Falvo, L. Grove, R. Stachura et al., "The opportunity loss of boarding admitted patients in the emergency department," Academic Emergency Medicine, vol. 14, no. 4, pp. 332-337, 2007.

[75] P. Krochmal and T. A. Riley, "Increased health care costs associated with ED overcrowding," American Journal of Emergency Medicine, vol. 12, no. 3, pp. 265-266, 1994.

[76] M. D. Bayley, J. S. Schwartz, F. S. Shofer et al., "The financial burden of emergency department congestion and hospital crowding for chest pain patients awaiting admission," Annals of Emergency Medicine, vol. 45, no. 2, pp. 110-117, 2005.

[77] P. L. Henneman, M. Lemanski, H. A. Smithline, A. Tomaszewski, and J. A. Mayforth, "Emergency department admissions are more profitable than non-emergency department admissions," Annals of Emergency Medicine, vol. 53, no. 2, pp. 249-255, 2009.

[78] J. M. Pines and J. D. Heckman, "Emergency department boarding and profit maximization for high-capacity hospitals: challenging conventional wisdom," Annals of Emergency Medicine, vol. 53, no. 2, pp. 256-258, 2009.
[79] J. Maa, "The waits that matter," The New England Journal of Medicine, vol. 364, no. 24, pp. 2279-2281, 2011.

[80] American College of Surgeons, History of Advanced Trauma Life Support, 2014, http://www.facs.org/trauma/atls/history .html.

[81] J. M. Brensilver, L. Smith, and C. S. Lyttle, "Impact of the Libby Zion case on graduate medical education in internal medicine," Mount Sinai Journal of Medicine, vol. 65, no. 4, pp. 296-300, 1998. 


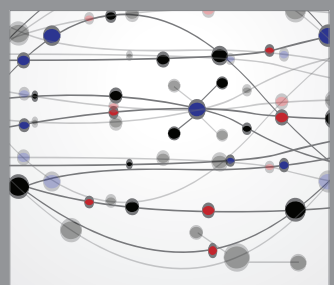

The Scientific World Journal
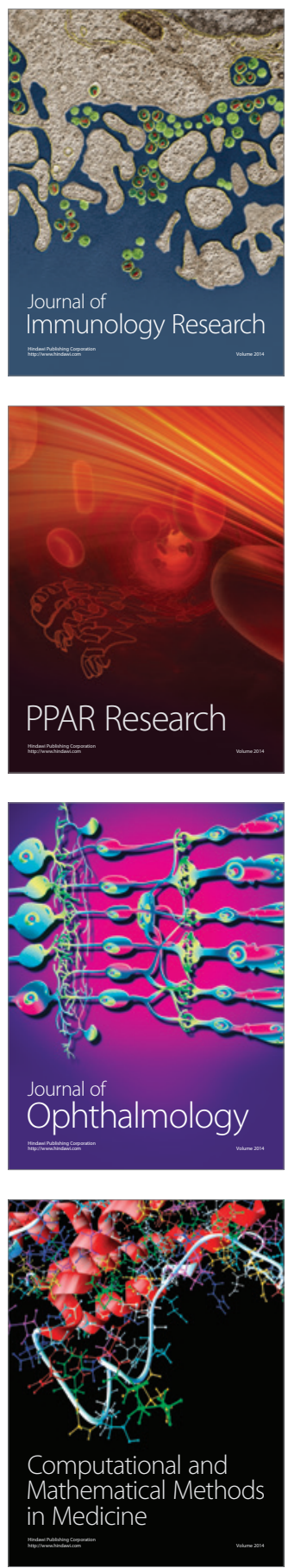

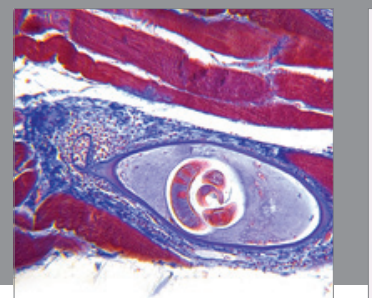

Gastroenterology

Research and Practice
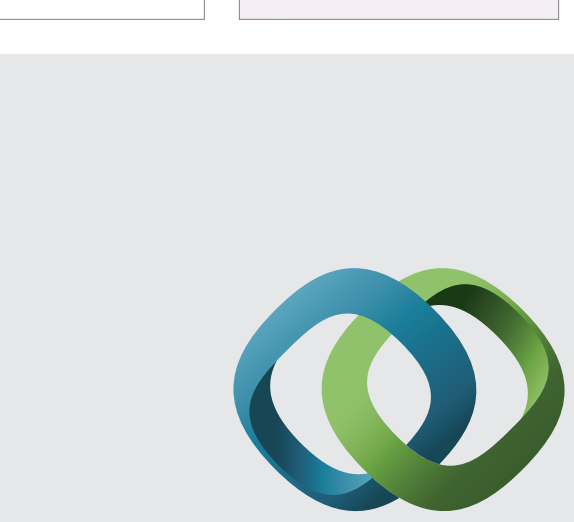

\section{Hindawi}

Submit your manuscripts at

http://www.hindawi.com
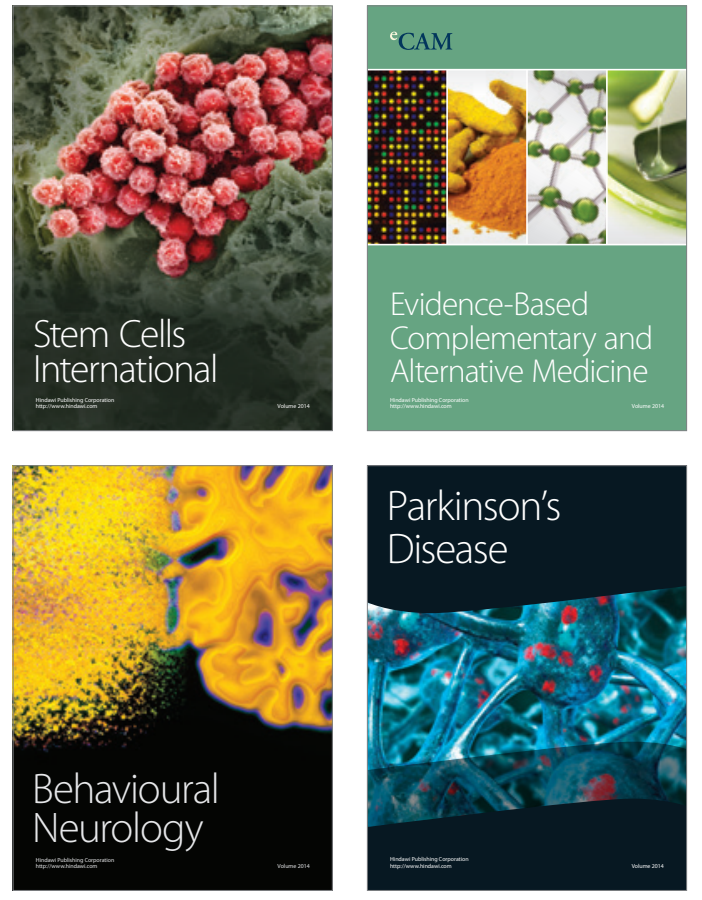
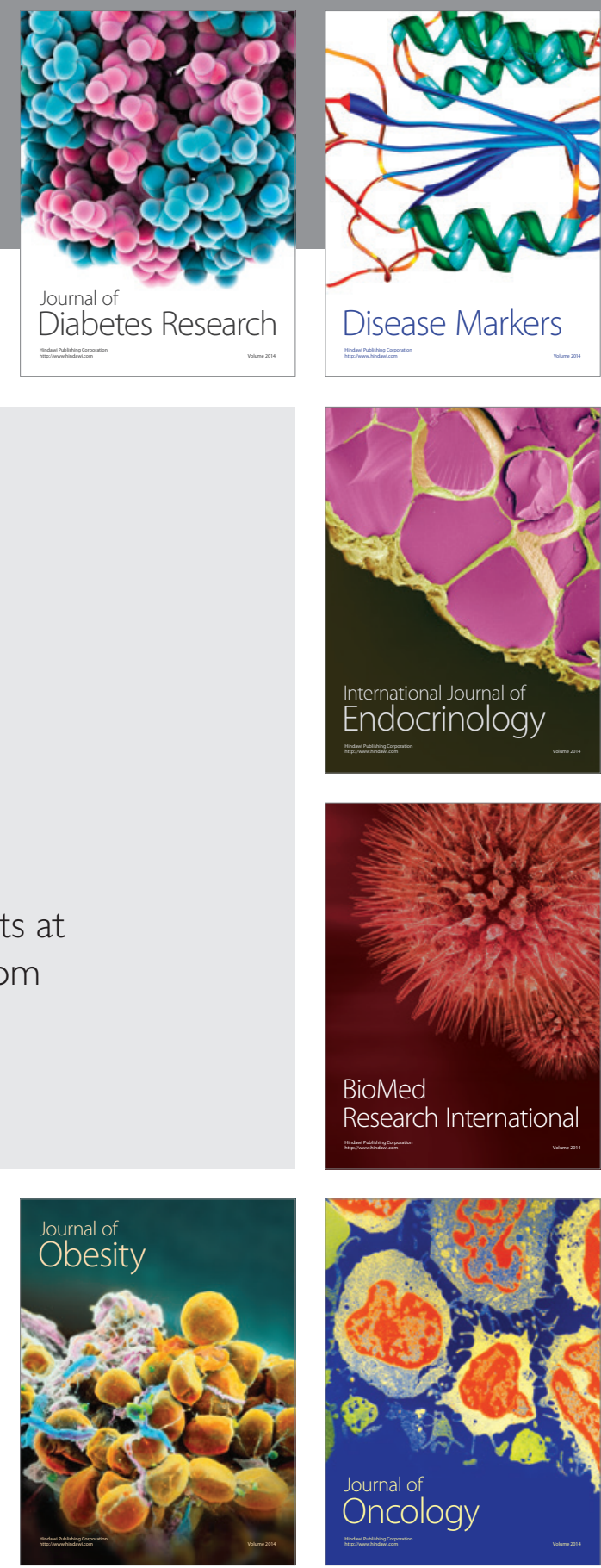

Disease Markers
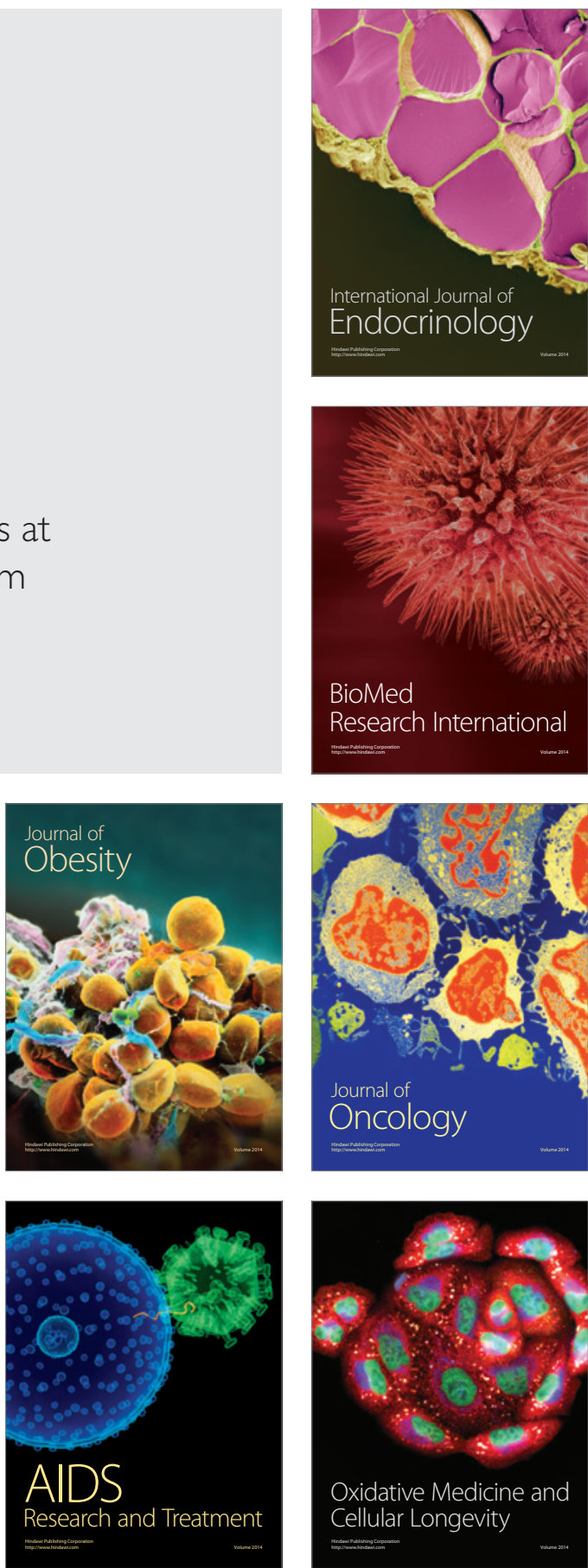